\title{
Generation of Multi-Scroll Chaotic Attractors from a Jerk Circuit with a Special Form of a Sine Function
}

\author{
Pengfei Ding ${ }^{1,2, *}$ and andiaoyi Feng ${ }^{1, *}$ \\ 1 School of Electronics and Information, Northwestern Polytechnical University, Xi'an 710072, China \\ 2 School of Electronics and Engineering, Xi'an University of Posts and Telecommunications, \\ Xi'an 710121, China \\ * Correspondence: dpf@xupt.edu.cn (P.D.); fengxiao@nwpu.edu.cn (X.F.)
}

Received: 31 March 2020; Accepted: 15 May 2020; Published: 19 May 2020

\begin{abstract}
A novel chaotic system for generating multi-scroll attractors based on a Jerk circuit using a special form of a sine function (SFSF) is proposed in this paper, and the SFSF is the product of a sine function and a sign function. Although there are infinite equilibrium points in this system, the scroll number of the generated chaotic attractors is certain under appropriate system parameters. The dynamical properties of the proposed chaotic system are studied through Lyapunov exponents, phase portraits, and bifurcation diagrams. It is found that the scroll number of the chaotic system in the left and right part of the $x-y$ plane can be determined arbitrarily by adjusting the values of the parameters in the SFSF, and the size of attractors is dominated by the frequency of the SFSF. Finally, an electronic circuit of the proposed chaotic system is implemented on Pspice, and the simulation results of electronic circuit are in agreement with the numerical ones.
\end{abstract}

Keywords: multi-scroll attractors; Jerk circuit; special form of a sine function; electronic circuit implementation

\section{Introduction}

Since Lorenz found the deterministic ordinary nonlinear differential equations has the characteristic of chaotic dynamics [1], chaotic system became a research focus around the world, and many researchers have been proposed many new three-dimensional chaotic systems, such as Chen system [2], Liu system [3], Lü system [4], Bao system [5], Rössler system [6], Sprott system [7], Liu-Chen system [8], Tigan system [9], Zhou system [10], Pham system [11]. With the deep research of chaos system, chaos system is widely used in engineering field. For example, weak signal detection [12-15], secure communication [16-18], random bit generator [19-21], and chaos-based image encryption [22-26]. As an important method for secure communication, chaos synchronization is studied by many researchers [16,18,27]. In [16], Lu et al. introduced the synchronization of a unified chaotic system via different method; in [18], Pano-Azucena, A.D. et al. gave a method of Hamiltonian-based controller for the synchronization of multi-directional multi-scroll chaotic attractors, and Voss, H.U. [27] presented the synchronization between two dissipative coupled Rössler systems. It is noteworthy that, Ravelo B. et al. [28,29] investigated a novel circuit theory of all-pass negative group delay (NGD) function, and the NGD function is implemented with unity direct chain feedback (UDCF) system, then, they investigated the feasibility of anticipating arbitrary signals from mechanical actuators with low-pass NGD function, and the study indicates the potential industrial applications of the NGD function in anomaly detections. Combined with the anomalous detection capability of NGD and the weak signal detection capability of chaotic system, a new method may be provided for industrial fault detection.

Suykens proposed a method for generating $\mathrm{n}$-double scroll attractors by adding more breakpoints at the first time [30]. Generally, with the increase of scroll number, the dynamic behaviors of the 
chaotic attractor become more complex. Hence, the multi-scroll chaotic system has attracted numerous researchers and different types of multi-scroll chaotic systems have been designed, such as 1-directional (1-D) multi-scroll chaotic systems [31,32], 2-directional (2-D) multi-scroll chaotic systems [33-37], 3-directional (3-D) multi-scroll chaotic system [38], and multi-directional multi-scroll chaotic system [39-41]. The realization of chaotic system based on FPGA has also been studied [42,43]. In three-dimensional differential equation, the general Jerk system has fewer terms, so the chaotic system based on general Jerk system has been extensively studied [44-48]. In practical application, the increase of the scroll numbers in chaotic systems is realized by increasing the number of equilibrium points. Since sine function has the characteristics of periodicity, many researchers designed multi-scroll chaotic system by modifying the expression of the sine functions or adding control function to chaotic system with sine function $[44,45,47,49-51]$.

In [44], Yu et al. introduced a modulating sine function to generate a scroll-nesting multi-scroll chaotic attractor. Yalçin [45] designed a multi-scroll chaotic system in a Jerk circuit by using a sine function. Ma et al. [47] proposed a multi-scroll chaotic system with a sine function and a feedback controlling function. Tang et al. [49] and Hu et al. [50] used different modified sine function for generating $\mathrm{n}$-scroll attractors. A sine function input is used in a hyper chaotic Liu system [51]. Motivated by these works, we proposed a multi-scroll chaotic system with a special form of a sine function (SFSF), the dynamical properties of this system are analyzed via Lyapunov exponents spectrum, chaotic phase portrait, and bifurcation diagram. Then the circuit diagram is designed for this system, the electronic circuit simulation results are consistent with the numerical ones.

The rest of the paper is organized as follows. In Section 2, the new multi-scroll chaotic system is designed based on SFSF in general Jerk circuit. The dynamical properties of the multi-scroll chaotic system are discussed in Section 3. In Section 4, the circuit implementation of the multi-scroll chaotic system is studied and simulated by Pspice. Finally, the conclusions are given in Section 5.

\section{A Novel Three-Dimensional Chaotic System Based on Jerk Circuits}

The so-called general Jerk circuits is described by

$$
\dddot{x}+\beta \ddot{x}+\gamma \dot{x}=f(x)
$$

In Equation (1), $\dot{x}$ is the derivative of the position called velocity, $\ddot{x}$ is the derivative of the velocity called acceleration, and $\dddot{x}$ is the derivative of the acceleration called Jerk. Let $\dot{x}=y, \dot{y}=z$, Equation (1) can be rewritten as follows.

$$
\left\{\begin{array}{l}
\dot{x}=y \\
\dot{y}=z \\
\dot{z}=-\gamma y-\beta z+f(x)
\end{array}\right.
$$

where, $\gamma, \beta$ are real numbers and $f(x)$ is the nonlinear function, which can be designed as sine function [44,45,47,49,52], hyperbolic tangent function [53,54], hyperbolic sine function [55], hyperbolic cosine function [56], piecewise linear function [57,58], hysteresis function series [41,59], saturated function series [60,61], and step function [62-64]. Based on Equation (2), a new multi-scroll chaotic system is proposed as follows.

$$
\left\{\begin{array}{l}
\dot{x}=10 y \\
\dot{y}=10 z \\
\dot{z}=10[-a y-c z-d f(x)]
\end{array}\right.
$$

where, the nonlinear function of $f(x)$ is a SFSF, which is different from the sine function in the literature and its expression is given by:

$$
f(x)=\left\{\begin{array}{l}
-\sin (2 \pi b x),-k_{n} / b \leq x \leq k_{p} / b \\
+\sin (2 \pi b x), x>k_{p} / b \\
+\sin (2 \pi b x), x<-k_{n} / b
\end{array}\right.
$$


In Equation (3), $a, c, d$ are real numbers, $k_{n}$ and $k_{p}$ are nonnegative integers, $x, y, z$ are state variables of system (3). The sine function $f(x)$ of Equation (4) is different from the sine function of $p(x)$ [44], $g(x)$ [45], and $h(x)$ [49], which is described as Equations (5)-(7).

$$
\begin{gathered}
p(x)=|a \sin (2 \pi b x)| \operatorname{sgn}(x)-x \\
g(x)=\sin (2 \pi b x) \\
h(x)=\left\{\begin{array}{l}
\frac{b \pi}{2 a}(x-2 a c), x \geq 2 a c \\
-b \sin \left(\frac{\pi x}{2 a}+d\right),-2 a c<x<2 a c \\
\frac{b \pi}{2 a}(x+2 a c), x \leq-2 a c
\end{array}\right.
\end{gathered}
$$

By setting the parameter value of $b$ as 0.25 , and the other parameters are set as $k_{p}=k_{n}=2$ in Equation (4), $a=6$ in Equation (5) and $a=1, c=5, d=0$ in Equation (7), the waveform of $f(x), p(x)$, $g(x)$, and $h(x)$ are depicted in Figure 1.

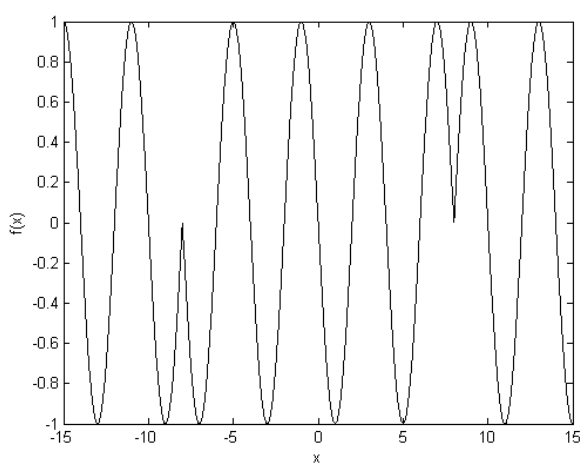

(a)

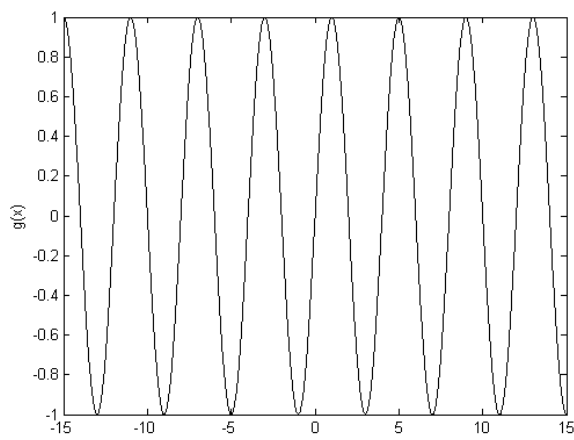

(c)

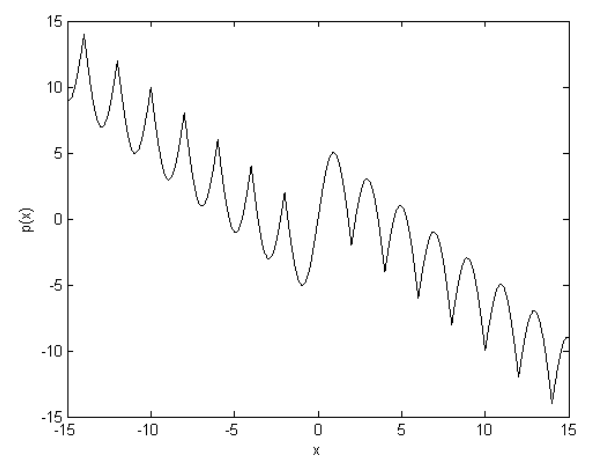

(b)

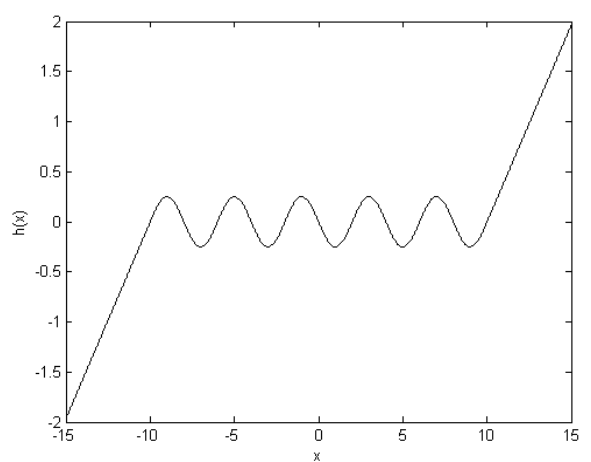

(d)

Figure 1. The waveform of different sine functions with $b=0.25$ : (a) $f(x)$ with $k_{p}=k_{n}=2$; (b) $p(x)$ with $a=6$; (c) $g(x) ;($ d) $h(x)$ with $a=1, c=5$, and $d=0$

From Figure 1, it can be seen that the number of the solutions of $p(x)=0$ and $h(x)=0$ are finite, but there are infinite solutions for $f(x)=0$ and $g(x)=0$. Yu et al. [44] designed a chaotic system with nonlinearity of $p(x)$ with $a=5$ and $b=1.27$ can generate 4 -scroll nesting chaotic attractors. A chaotic system with nonlinearity of $h(x)$ [49] can generate $(c+1)$-scroll chaotic attractors. Yalçin et al. [45] showed that the scroll number of the chaotic system with $g(x)$ is increased with longer simulation time. $f(x)=0$ as the same as $g(x)=0$ has infinite solutions, however, we found that the scroll number generated by system (3) with the nonlinearity of $f(x)$ does not vary with simulation time under suitable parameters. As an example, the system parameters are selected as $a=c=0.3, b=d=0.5$, $k_{p}=k_{n}=3$, chaotic system (3) with $\left(x_{0}, y_{0}, z_{0}\right)=(0.1,0.1,0.1)$ can generate 6-scroll attractors with different simulation duration, as shown in Figure $2 \mathrm{a}, \mathrm{b}$. 


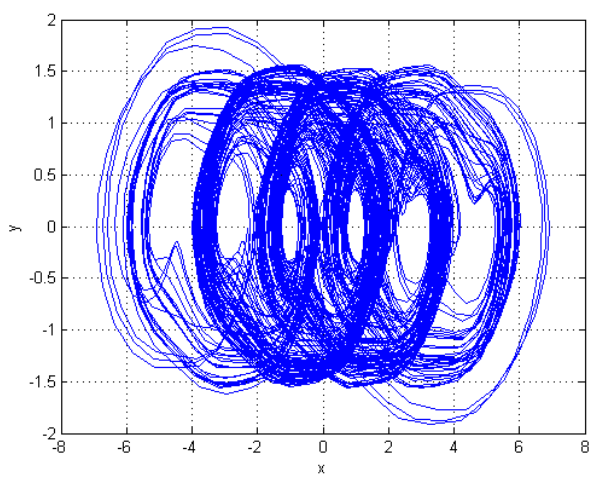

(a)

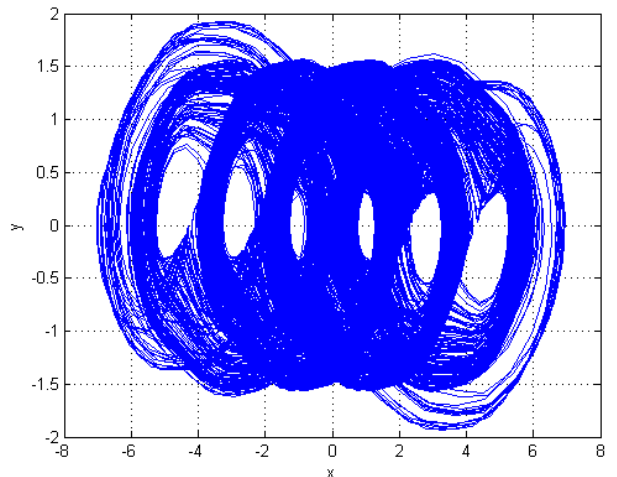

(b)

Figure 2. The 6-scroll chaotic attractor is generated by system (3) with $a=c=0.3, b=d=0.5$, $k_{p}=k_{n}=3$, and $\left(x_{0}, y_{0}, z_{0}\right)=(0.1,0.1,0.1):(\mathbf{a})$ simulation time selected as 5000 time units and (b) simulation time selected as 20,000 time units.

As seen from Figure 2a,b, the generated scroll number by system (3) with $f(x)$ does not vary with the simulation time. It is quite different from the chaotic system with $g(x)$ [45], which has varied scroll number with different simulation time.

\section{Theoretical Analysis of the Chaotic System}

\subsection{Dissipativity of the New Chaotic System}

The dissipativity of system (3) is described by:

$$
\nabla V=\frac{\partial x}{\partial x}+\frac{\partial y}{\partial y}+\frac{\partial z}{\partial z}=-10 c
$$

In Equation (8), $V$ is a vector, and $x, y, z \in R, c$ is constant, when $c>0$, system (3) is dissipative, and the expression of the exponential contraction is:

$$
\frac{d V}{d t}=e^{-10 c}
$$

From Equation (9), a volume element for initial $V(0)$ is contracted by the rate of $e^{-10 c t}$ at time $t$, so each volume element's trajectory of system (3) shrinks to zero when the time $t$ gets close to $\infty$. As a result, all trajectories of system will be converged to a point set with zero volume.

\subsection{Equilibrium Points of the Chaotic System}

In order to obtain the equilibrium points of system (3), setting the right hand side of system (3) to zero, then one gets equilibrium points given by

$$
\left\{\begin{array}{l}
y=0 \\
z=0 \\
f(x)=0
\end{array}\right.
$$

From Equations (10), the equilibrium points can be calculated by $\left(E_{x, n}, 0,0\right)$, where $E_{x, n}=\mathrm{n} / 2 \mathrm{~b}$, $\mathrm{n}=0, \pm 1, \pm 2, \pm 3, \ldots$, obviously, the distance between two adjacent points is $1 / 2 \mathrm{~b}$. Assuming $T_{1}=T / 2=1 /(2 b)$, the equilibrium points are $Q_{0}(0,0,0), Q_{1}\left(T_{1}, 0,0\right), Q_{1}\left(-T_{1}, 0,0\right), Q_{2}\left(2 T_{1}, 0,0\right)$, $Q_{-2}\left(-2 T_{1}, 0,0\right), \ldots, Q_{m}\left(m T_{1}, 0,0\right), Q_{-m}\left(-m T_{1}, 0,0\right)$, and $m$ is a nonnegative integer. 
Equation (10) combined with Equations (3) and (4), the equilibrium points of system (3) exist in five subspaces, which are described as follows:

$$
\begin{aligned}
& E_{1}=\left\{Q_{m_{1}}\left(m_{1} T_{1}, 0,0\right) \mid-2 k_{n}<m_{1}<2 k_{p}\right\}, \\
& E_{2}=\left\{Q_{m_{2}}\left(m_{2} T_{1}, 0,0\right) \mid m_{2}>2 k_{p}\right\}, \\
& E_{3}=\left\{Q_{m_{3}}\left(m_{3} T_{1}, 0,0\right) \mid m_{3}<-2 k_{n}\right\}, \\
& E_{4}=\left\{Q_{m_{4}}\left(m_{4} T_{1}, 0,0\right) \mid m_{4}=2 k_{p}\right\}, \\
& E_{5}=\left\{Q_{m_{5}}\left(m_{5} T_{1}, 0,0\right) \mid m_{5}=-2 k_{n}\right\},
\end{aligned}
$$

where, $T_{1}=T / 2=1 /(2 b), k_{n}$ and $k_{p}$ are the system parameter in Equation (4). Let $a=c=\sigma=0.3$ and $b=d=0.5$, the equilibrium points of system (3) are analyzed as follows:

(i) $\quad Q_{m}\left(m T_{1}, 0,0\right)=Q_{m_{11}}\left(m_{11} T_{1}, 0,0\right) \in E_{1}$ and $m_{11}$ is an even integer

The Jacobian matrix of system (3) at the equilibrium points of $Q_{m_{11}}\left(m_{11} T_{1}, 0,0\right)$ can be obtained as

$$
J_{1}=10\left(\begin{array}{ccc}
0 & 1 & 0 \\
0 & 0 & 1 \\
-d f^{\prime}(x) & -\sigma & -\sigma
\end{array}\right)=10\left(\begin{array}{ccc}
0 & 1 & 0 \\
0 & 0 & 1 \\
d \pi & -\sigma & -\sigma
\end{array}\right)=10\left(\begin{array}{ccc}
0 & 1 & 0 \\
0 & 0 & 1 \\
0.5 \pi & -0.3 & -0.3
\end{array}\right)
$$

By linearizing Equation (3) at $Q_{m_{11}}\left(m_{11} T_{1}, 0,0\right)$, the characteristic equation of the Jacobian matrix (11) for system (3) is described as follows:

$$
\left|\lambda I-J_{1}\right|=\lambda^{3}+\sigma \lambda^{2}+\sigma \lambda-d \pi=\lambda^{3}+0.3 \lambda^{2}+0.3 \lambda-0.5 \pi=0
$$

where, $I$ is unit matrix, and the eigenvalues of Equation (12) are $\lambda_{1}=0.799, \lambda_{2}=-0.550+0.936 i$, $\lambda_{3}=-0.550-0.936 i$, so the equilibrium points $Q_{m_{11}}\left(m_{11} T_{1}, 0,0\right)$ are saddle points of index 1 .

(ii) $Q_{m}\left(m T_{1}, 0,0\right)=Q_{m_{12}}\left(m_{12} T_{1}, 0,0\right) \in E_{1}$ and $m_{12}$ is an odd integer

At the equilibrium points $Q_{m_{12}}\left(m_{12} T_{1}, 0,0\right)$, the Jacobian matrix of system (3) is expressed as

$$
J_{2}=10\left(\begin{array}{ccc}
0 & 1 & 0 \\
0 & 0 & 1 \\
-d f^{\prime}(x) & -\sigma & -\sigma
\end{array}\right)=10\left(\begin{array}{ccc}
0 & 1 & 0 \\
0 & 0 & 1 \\
-d \pi & -\sigma & -\sigma
\end{array}\right)=10\left(\begin{array}{ccc}
0 & 1 & 0 \\
0 & 0 & 1 \\
-0.5 \pi & -0.3 & -0.3
\end{array}\right)
$$

By linearizing Equation (3) at $Q_{m_{12}}\left(m_{12} T_{1}, 0,0\right)$, the characteristic equation of the Jacobian matrix (13) for system (3) is expressed as follows:

$$
\left|\lambda I-J_{2}\right|=\lambda^{3}+\sigma \lambda^{2}+\sigma \lambda+d \pi=\lambda^{3}+0.3 \lambda^{2}+0.3 \lambda+0.5 \pi=0
$$

The eigenvalues of Equation (14) are $\lambda_{1}=-0.978, \lambda_{2}=0.339+0.921 i, \lambda_{3}=0.339-0.921 i$, So the equilibrium point $Q_{m_{12}}\left(m_{12} T_{1}, 0,0\right)$ are saddle points of index 2 .

For subspaces of $E_{2}$ and $E_{3}$, the analysis are similar to the subspace of $E_{1}$, we only give the results as follows:

(iii) $Q_{m_{21}}\left(m_{21} T_{1}, 0,0\right) \in E_{2}$ and $m_{21}$ is an even integer, $Q_{m_{21}}\left(m_{21} T_{1}, 0,0\right)$ are saddle points of index 2 .

(iv) $Q_{m_{22}}\left(m_{22} T_{1}, 0,0\right) \in E_{2}$ and $m_{22}$ is an odd integer, $Q_{m_{22}}\left(m_{22} T_{1}, 0,0\right)$ are saddle points of index 1 .

(v) $Q_{m_{31}}\left(m_{31} T_{1}, 0,0\right) \in E_{3}$ and $m_{31}$ is an even integer, $Q_{m_{31}}\left(m_{31} T_{1}, 0,0\right)$ are saddle points of index 2 .

(vi) $Q_{m_{32}}\left(m_{32} T_{1}, 0,0\right) \in E_{3}$ and $m_{32}$ is an odd integer, $Q_{m_{32}}\left(m_{32} T_{1}, 0,0\right)$ are saddle points of index 1 .

As to equilibrium point $Q_{m_{4}}\left(m_{4} T_{1}, 0,0\right) \in E_{4}$ and $Q_{m_{5}}\left(m_{5} T_{1}, 0,0\right) \in E_{5}$, from Equation (3) and Figure $1 \mathrm{a}$, it can be seen that $Q_{m_{4}}\left(m_{4} T_{1}, 0,0\right)$ and $Q_{m_{5}}\left(m_{5} T_{1}, 0,0\right)$ are not saddle points. 
As an example, the distribution of the saddle points of system (3) with $b=0.25$ and $k_{p}=k_{n}=2$ on $x$ coordinate axis is shown in Figure 3.

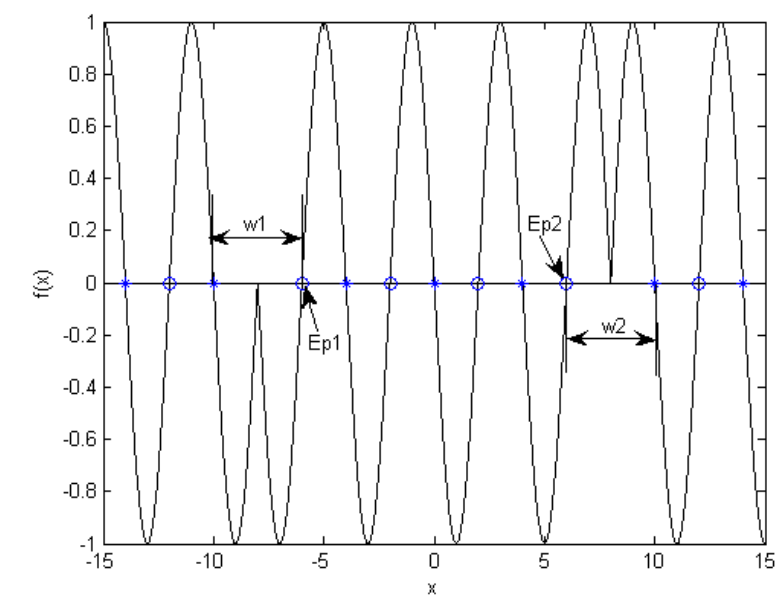

Figure 3. The distribution of the saddle points of system (3) with $b=0.25$ and $k_{p}=k_{n}=2$ on $\mathrm{x}$ coordinate axis.

In Figure 3, "o" represents the saddle point of index 2, and "** represents the saddle point of index 1. Ep1 is the equilibrium point of $Q\left(\left(-2 k_{n}+1\right) \tau_{1}, 0,0\right)$, while Ep2 is the equilibrium point of $Q\left(\left(2 k_{p}-1\right) \tau_{1}, 0,0\right)$, where $k_{n}$ and $k_{p}$ are the system parameters in Equation (4) and they are nonnegative integer, $\tau_{1}=1 /(2 b)=2$ is equals to half of the period of the sine function in the SFSF, which is described in Equation (4). Figure 3 shows two special saddle points of index 2 of Ep1 and Ep2, the distance from Ep1 to its left neighboring saddle point of index 1 and its right one are $w 1=1 / b=2 \tau_{1}$ and $\tau_{1}$, respectively. Similarly, the distance from Ep2 to its left neighboring saddle point of index 1 and its right one are $\tau_{1}$ and $w 2=1 / b=2 \tau_{1}$, respectively. Except to the saddle points Ep1 and Ep2, the distance from the saddle points of index 2 of system (3) to its left neighboring saddle point of index 1 is equal to the distance from it to its right one.

Yu et al. [44] indicated that chaotic trajectories rotate around the saddle points of index 2 to form a scroll, and that trajectories move away from the saddle points of index 1 to its neighboring scrolls to form the whole multi-scroll chaotic attractor. Moreover, Wang et al. [65] pointed out that in multi-scroll chaotic systems, scrolls are generated only around the saddle points of index 2 , and neighboring scrolls are connected by the saddle points of index 1 . The width between two neighboring scrolls of the chaotic systems with saw-tooth waves and staircase nonlinear functions are given by Yu et al. [44] and Sánchez-López [66], respectively. Similarly, the width between two neighboring scrolls of this introduced system is determined by the distance between two adjacent saddle points of index 2 . The width of the scrolls (except the outside edge scroll), which generate by this introduced chaotic system is given as follows:

$$
w=1 / b .
$$

Consequently, with the appropriate system parameters, the generated scroll by system (3) around Ep1 cannot move to its left neighboring saddle point of index 1, thus avoid the generation of new scroll in the left side of Ep1. In exactly the same way, no new scrolls can be generated in the right side of Ep2. As a result, if the system parameter is selected appropriately, system (3) with nonlinearity of Equation (4) can generate fixed number scroll chaotic attractors.

\subsection{Lyapunov Exponents Spectrum and Bifurcation Diagram}

When $a=c=0.3, b=0.25, k_{p}=k_{n}=3$ at the initial state of $\left(x_{0}, y_{0}, z_{0}\right)=(0.1,0.1,0.1)$, the parameter $d$ varies from 0 to 3 with step size 0.01 ; the Lyapunov exponents spectrum of system (3) 
can be obtained as shown in Figure 4a. By using the same parameter values in bifurcation study, the bifurcation diagram is presented in Figure 4 b.

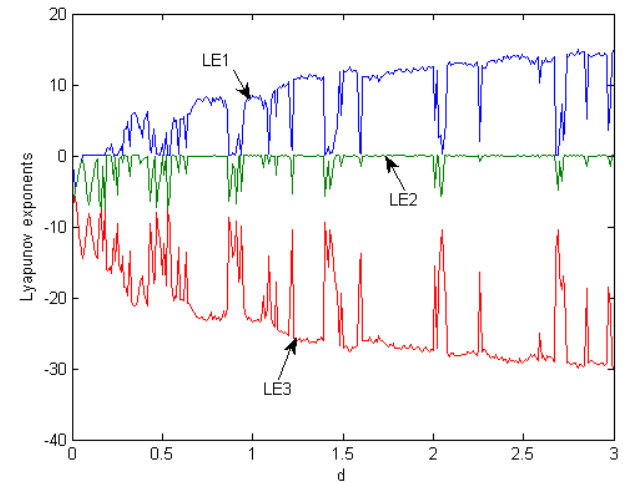

(a)

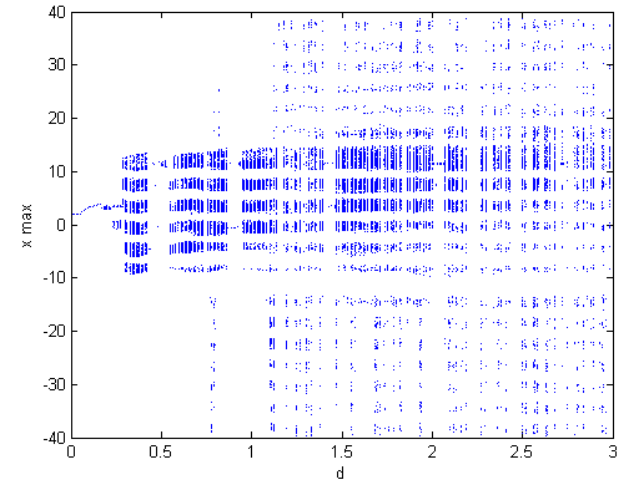

(b)

Figure 4. Lyapunov exponents spectrum and bifurcation diagram of system (3) with $a=c=0.3$, $b=0.25, k_{p}=k_{n}=3,\left(x_{0}, y_{0}, z_{0}\right)=(0.1,0.1,0.1)$ and $d \in[0,3]$ : (a) Lyapunov exponents spectrum; and $(\mathbf{b})$ bifurcation diagram.

From the numerical results in Figure 4, it can be seen that when $d \in[0,0.05], \lambda_{L E 1}<0, \lambda_{L E 2}<0$, $\lambda_{L E 3}<0$, system (3) is stable. When $d \in[0.06,0.15] \cup[0.43,0.54], \lambda_{L E 1}=0, \lambda_{L E 2}<0, \lambda_{L E 3}<0$, system (3) is in cycle state. When $d \in[0.16,0.42], \lambda_{L E 1}>0, \lambda_{L E 2}=0, \lambda_{L E 3}<0$, system (3) is in chaotic. In the region of $d \in[0.55,3.0]$, system (3) is in chaotic state in most of the region, and a few in cycle state.

\subsection{Chaotic Phase Diagram}

For further study the dynamics of the proposed system (3), the phase portraits of the new system (3) with parameter values of $a=c=0.3, b=0.25, k_{p}=k_{n}=3,\left(x_{0}, y_{0}, z_{0}\right)=(0.1,0.1,0.1)$ are presented as follows.

Let $d=0.03$, the phase portraits of the system are given in Figure 5. Figure 5a-d give the phase portraits in $x-y-z$ plane, in $x-y$ plane, $x-z$ plane, and $y-z$ plane respectively. From Figure $5 a-d$, it can be seen that system (3) is in stable, that is in agreement with the Lyapunov exponents spectrum and bifurcation diagram.

Let $d=0.12$, the phase portraits of system (3) in $x-y-z$ plane, in $x-y$ plane, $x-z$ plane, and $y-z$ plane are shown in Figure $6 a-d$, respectively. Figure $6 a-d$ indicate that system (3) is in cycle state, that agrees with the Lyapunov exponents spectrum and bifurcation diagram in Figure 4.

Let $d=0.35$, the phase portraits of system (3) in $x-y-z$ plane, in $x-y$ plane, $x-z$ plane, and $y-z$ plane are shown in Figure 7. Figure 7a-d shows that system (3) is in chaotic state, that is in accordance with the Lyapunov exponents spectrum and bifurcation diagram in Figure 4. From Figure 7b, it can be seen that system (3) can generate 6-scroll chaotic attractors.

Let $d=2.5$, the phase portraits of system (3) in $x-y-z$ plane, in $x-y$ plane, $x-z$ plane, and $y-z$ plane are shown in Figure 8. From Figure $8 \mathrm{a}-\mathrm{d}$, it can be concluded that system (3) is in chaotic state, that is consistent with the Lyapunov exponents spectrum and bifurcation diagram in Figure 4. However, the number of scrolls generated by system (3) is far more than 6, which is quite different from Figure 7.

In order to verify the relationship between the parameters $k_{p}, k_{n}$, the scroll number of the chaotic attractors is generated by system (3). Let $a=c=0.3, b=0.25, d=0.35$, and $\left(x_{0}, y_{0}, z_{0}\right)=(0.1,0.1,0.1)$, and varying the parameter values of $k_{p}$ and $k_{n}$, the phase portraits in $x-y$ plane are shown in Figure 9.

From Figure 9, it can be seen that the scrolls of the chaotic attractor can be distributed both in the left and right sides of the $x-y$ plane or only in the right side of the $x-y$ plane. Furthermore, the parameters $k_{p}$ and $k_{n}$ control the scroll number in the left and right side of the $\mathrm{x}-\mathrm{y}$ plane. Consequently, 
the scrolls of the chaotic attractor generated by system (3) can be distributed in both left and right parts or only in the left or right part of the $x-y$ plane.

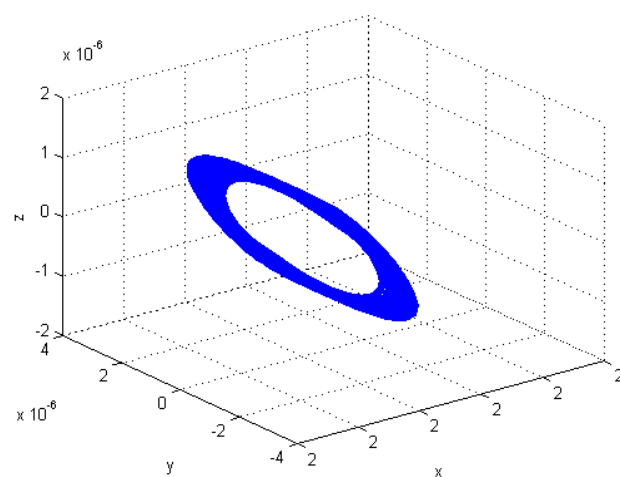

(a)

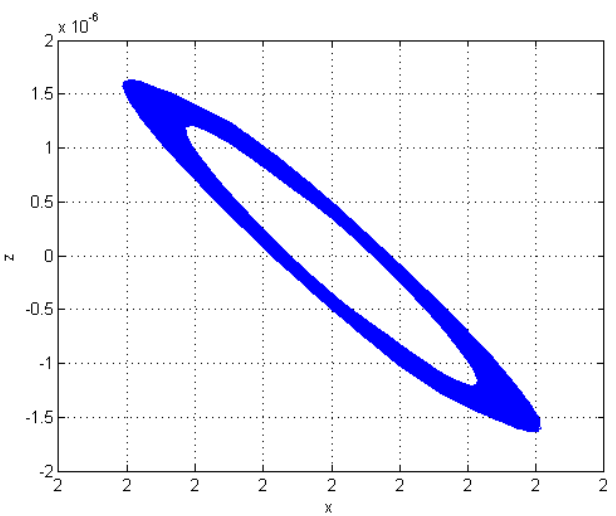

(c)

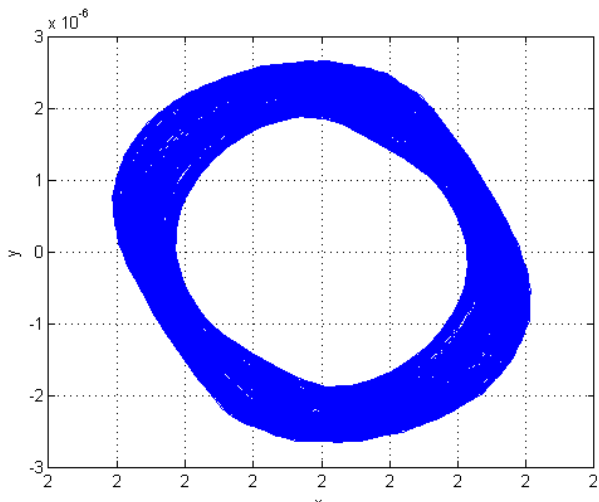

(b)

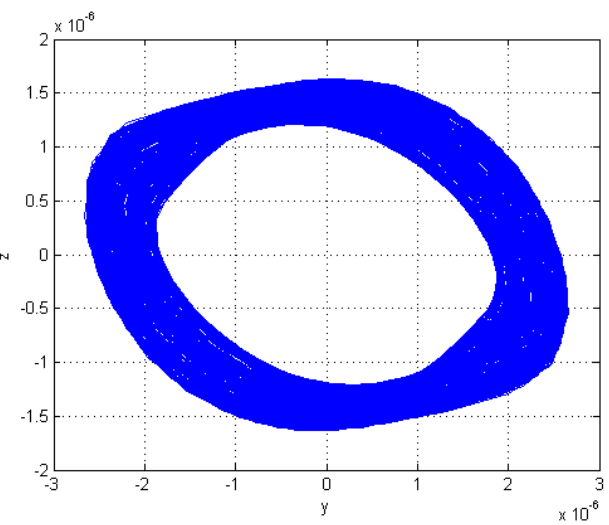

(d)

Figure 5. The phase portrait of system (3) with $\left(x_{0}, y_{0}, z_{0}\right)=(0.1,0.1,0.1), a=c=0.3, b=0.25$, $k_{p}=k_{n}=3, d=0.02$ : (a) $\mathrm{x}-\mathrm{y}-\mathrm{z}$ plane; (b) $\mathrm{x}$-y plane; (c) $\mathrm{x}$-z plane; and (d) $\mathrm{y}$-z plane.

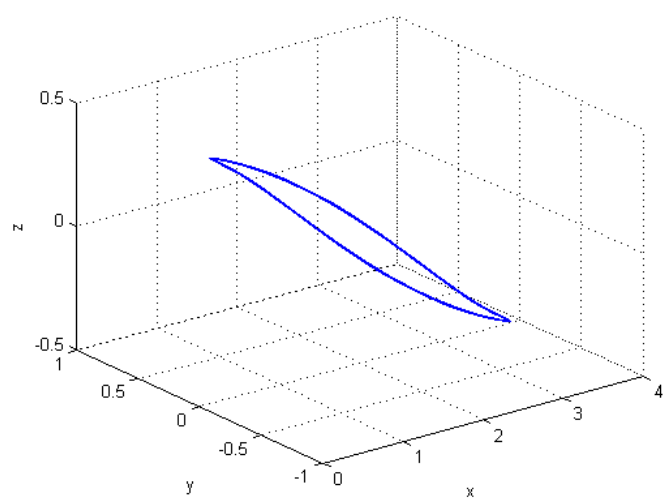

(a)

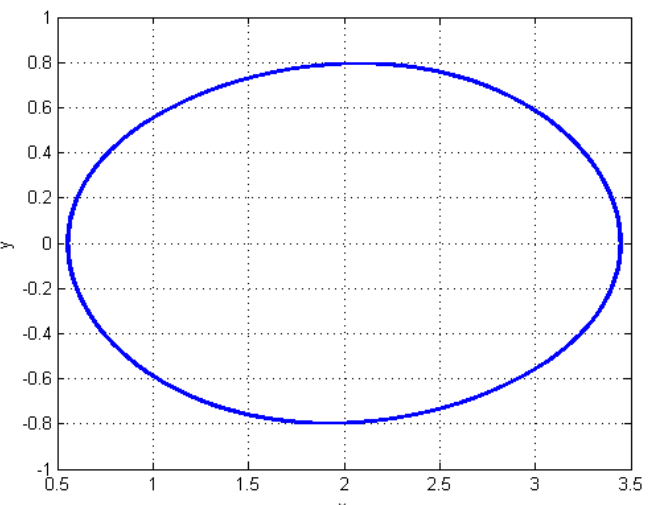

(b)

Figure 6. Cont. 


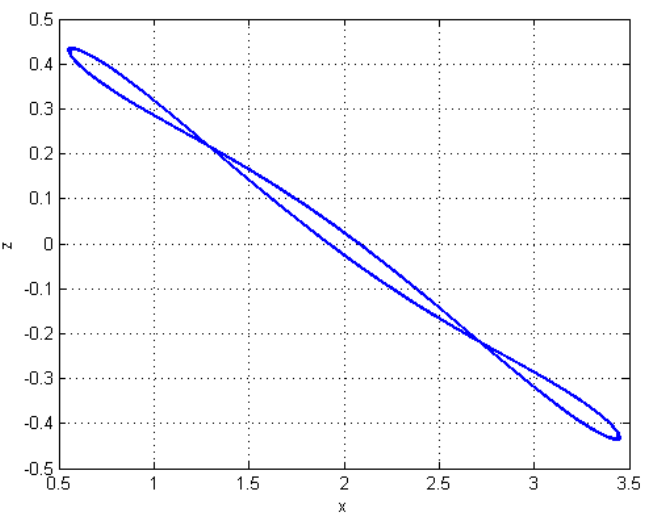

(c)

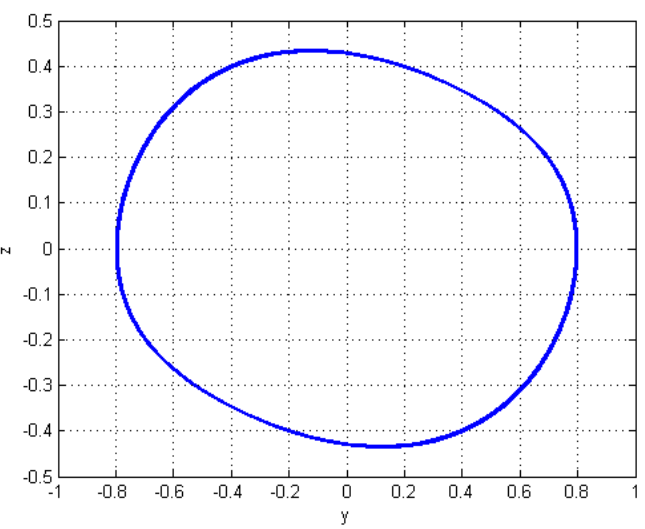

(d)

Figure 6. The phase portrait of system (3) with $\left(x_{0}, y_{0}, z_{0}\right)=(0.1,0.1,0.1), a=c=0.3, b=0.25$, $k_{p}=k_{n}=3, d=0.12$ : (a) $\mathrm{x}-\mathrm{y}-\mathrm{z}$ plane; (b) $\mathrm{x}$-y plane; (c) $\mathrm{x}-\mathrm{z}$ plane; and (d) $\mathrm{y}$-z plane.

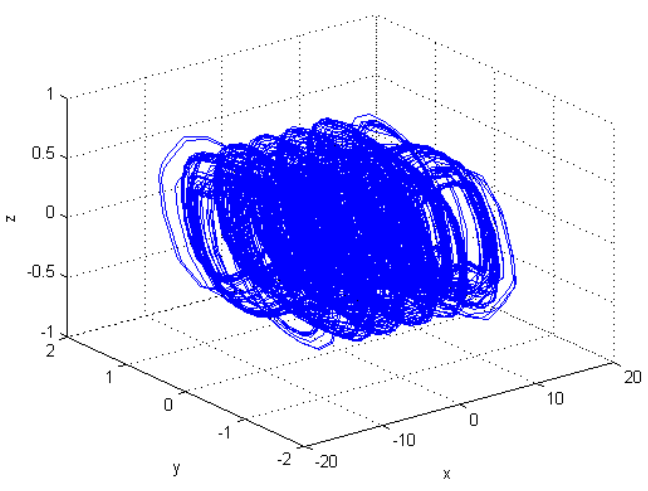

(a)

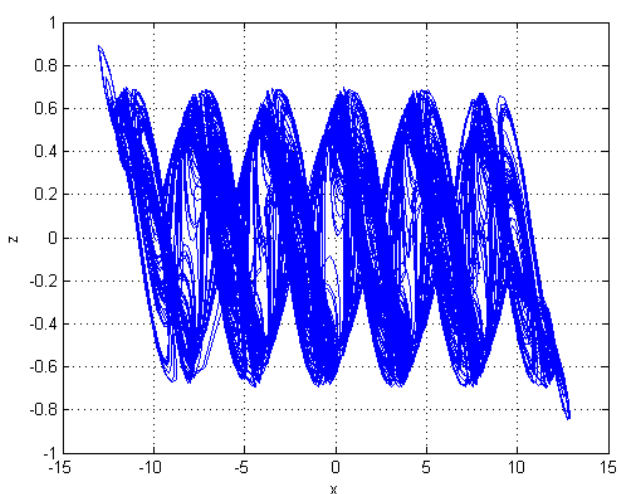

(c)

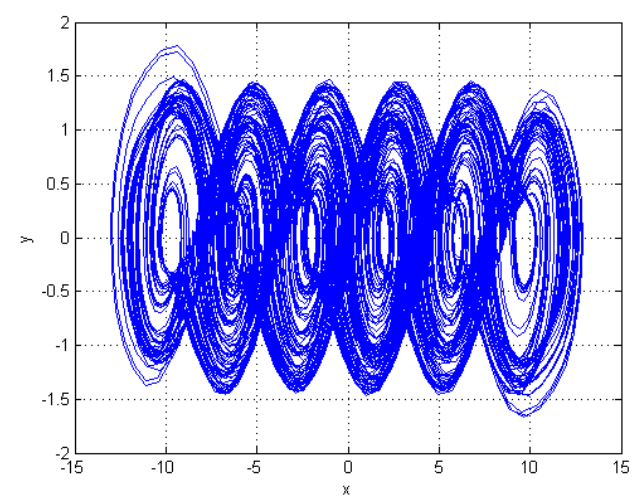

(b)

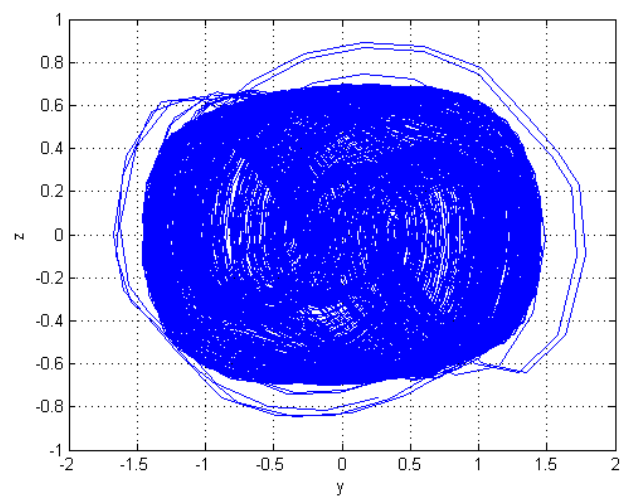

(d)

Figure 7. The phase portrait of system (3) with $\left(x_{0}, y_{0}, z_{0}\right)=(0.1,0.1,0.1), a=c=0.3, b=0.25$, $k_{p}=k_{n}=3$ and $d=0.35$ : (a) $\mathrm{x}-\mathrm{y}-\mathrm{z}$ plane; (b) x-y plane; (c) x-z plane; and (d) y-z plane. 


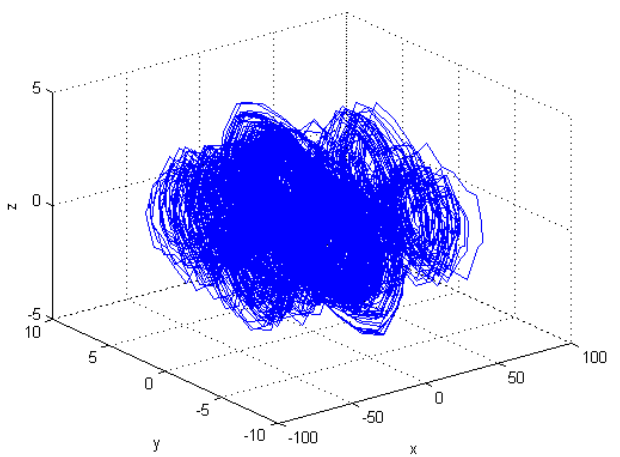

(a)

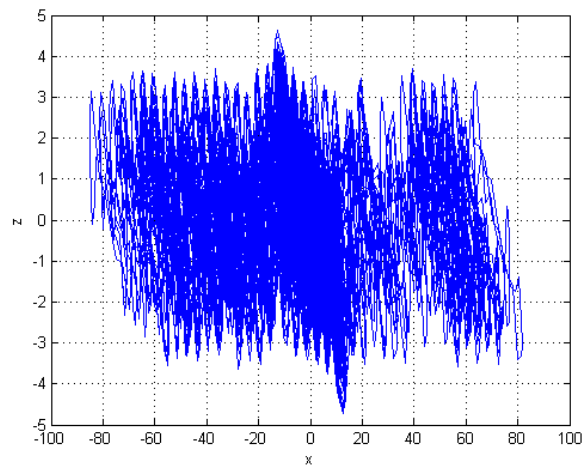

(c)

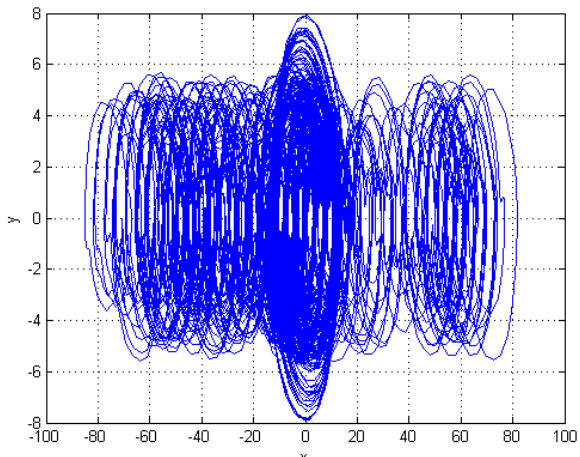

(b)

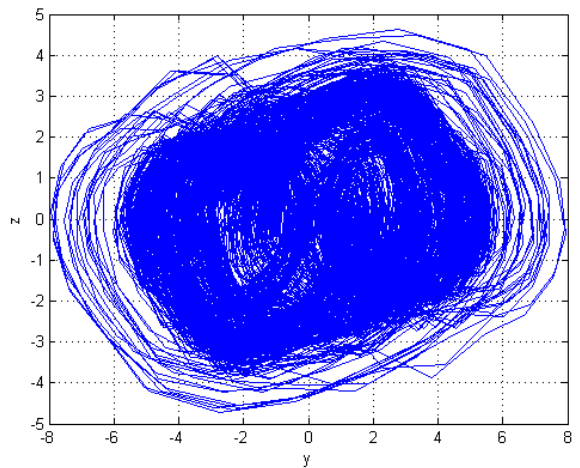

(d)

Figure 8. The phase portrait of system (3) with $\left(x_{0}, y_{0}, z_{0}\right)=(0.1,0.1,0.1), a=c=0.3, b=0.25$, $k_{p}=k_{n}=3$ and $d=2.5$ : (a) $\mathrm{x}-\mathrm{y}-\mathrm{z}$ plane; (b) $\mathrm{x}-\mathrm{y}$ plane; (c) $\mathrm{x}-\mathrm{z}$ plane; and (d) y-z plane.

According to Figures 5-9, we can obtain the following conclusions:

(i) The new system (3) with the SFSF can be in stable, cyclic, or chaotic state.

(ii) With suitable parameter values, the new system (3) can generate multi-scroll chaotic attractors (i.e., 3-scroll, 4-scroll, 5-scroll, and 6-scroll, which are displayed in Figures 9 and 10).

(iii) The chaotic attractors generated by the new system (3) can be distributed in the left side or/and right side of the $x-y$ plane.

(iv) The scroll number in the left and right parts of the $x-y$ plane is determined by the control parameters $k_{p}$ and $k_{n}$, which are the system parameters in the nonlinear function (4).

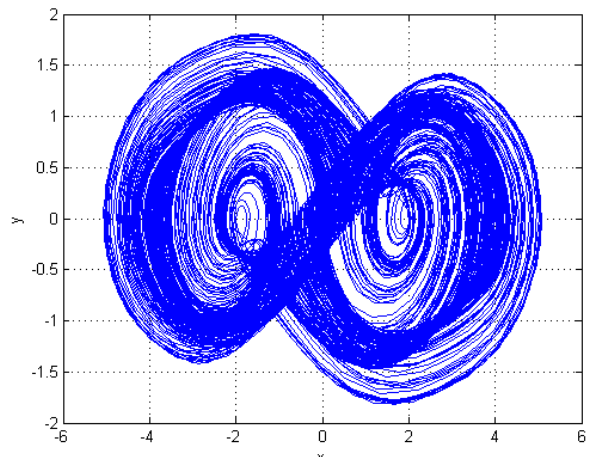

(a)

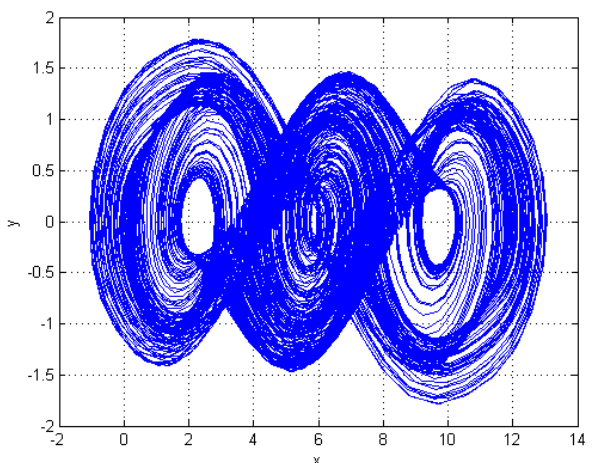

(b)

Figure 9. Cont. 


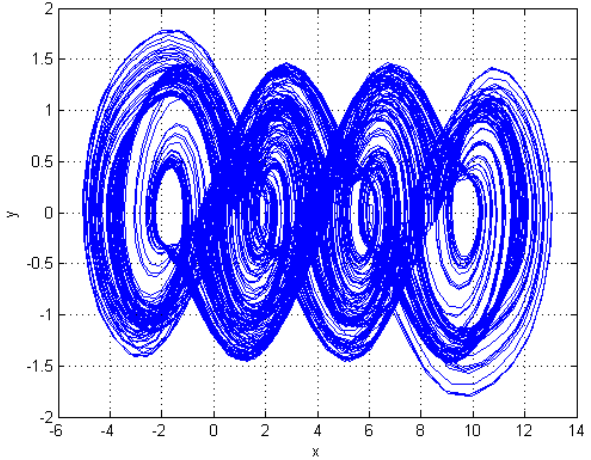

(c)

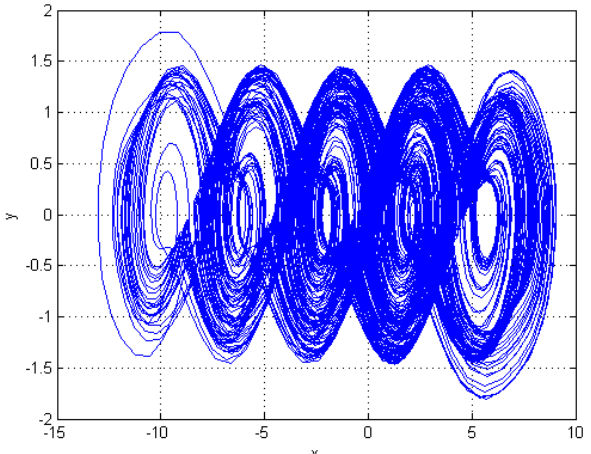

(d)

Figure 9. The phase portrait of system (3) in $x-y$ plane with $\left(x_{0}, y_{0}, z_{0}\right)=(0.1,0.1,0.1), a=c=0.3$, $b=0.25, d=0.35$ : (a) $k_{p}=k_{n}=1 ;(\mathbf{b}) k_{p}=3, k_{n}=0 ;$ (c) $k_{p}=2, k_{n}=2$; and (d) $k_{p}=2, k_{n}=3$.

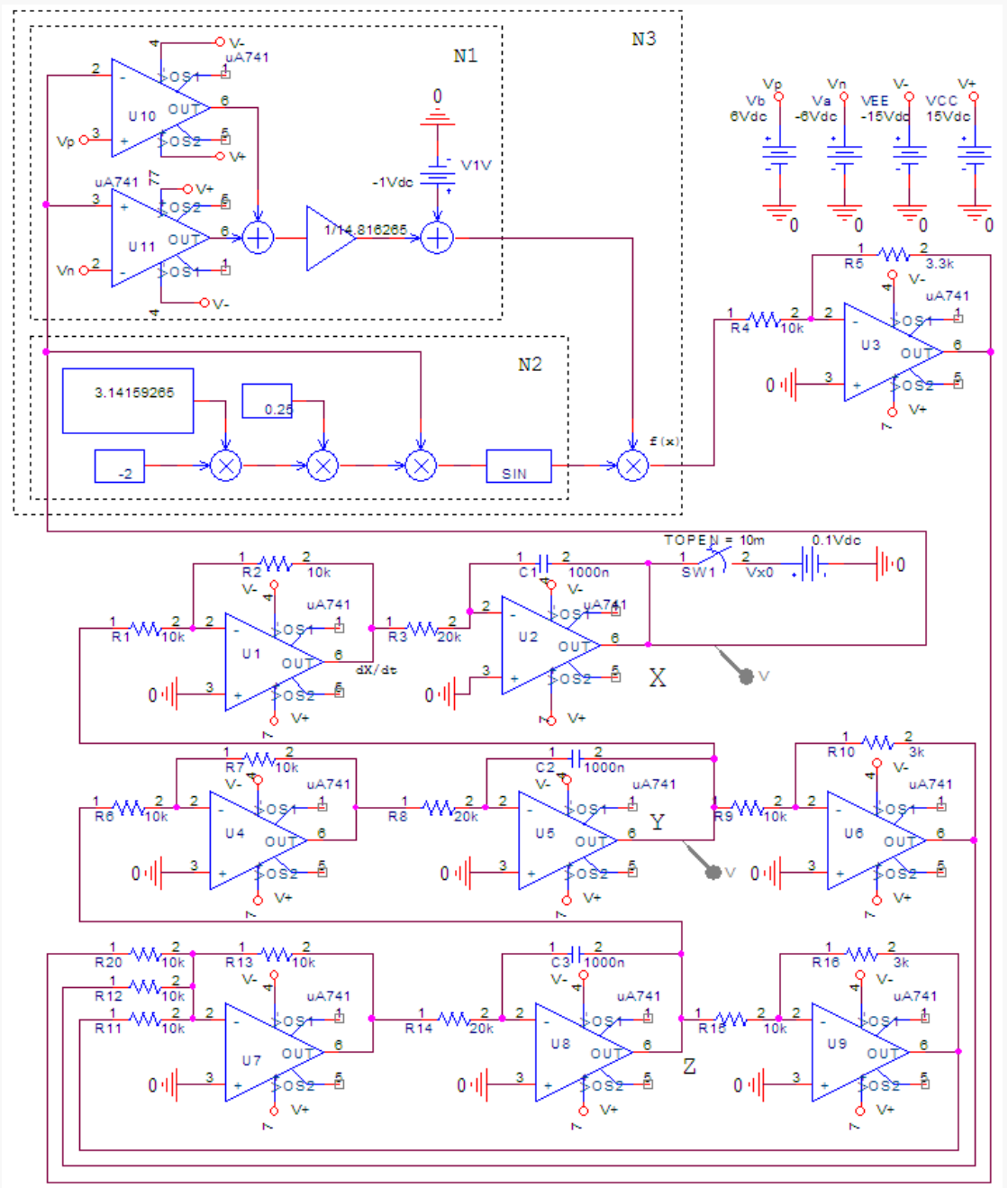

Figure 10. The circuit diagram of system (3).

\section{Electronic Circuit Implementation on Pspice}

In this section, an electronic circuit is designed on Pspice to confirm system (3) dynamical behaviors with numerical simulations. The schematic diagram of new chaotic system (3) is shown in Figure 10. Operational amplifier of type uA741 with power supply of $\pm 15 \mathrm{~V}$ is used to realize addition, subtraction, and integration of signal, the nonlinear function is realized by the part N3 of schematic diagram, which 
consists of part N1 and N2 in Figure 10. Circuit part N2 is a sine function generator, which contains a packaged arithmetic unit in Pspice.

Through analysis, we found that Equation (4) can be rewritten as follows.

$$
f(x)=-\sin (2 \pi b x) H\left(x, v_{n}, v_{p}\right)
$$

where

$$
H\left(x, v_{n}, v_{p}\right)=\operatorname{sign}\left(x-v_{n}\right)-\operatorname{sign}\left(x-v_{p}\right)-1, v_{n}=-k_{n} / b, v_{p}=k_{p} / b
$$

In Equation (17), $\operatorname{sign}(x)$ is a signal function as $\operatorname{sign}(x)=1$ for $x>0, \operatorname{sign}(x)=0$ for $x=0$ and $\operatorname{sign}(x)=-1$ for $x<0, k_{n}$ and $k_{p}$ are non-negative integers. Let $v_{n}=-4, v_{p}=4$ and $b=0.25$, the simulation results of $H\left(x, v_{n}, v_{p}\right)$ and $f(x)$ by Matlab are shown in Figure 11. The circuits of $H\left(x, v_{n}, v_{p}\right)$ and $f(x)$ are realized by the part N2 and part N3 in Figure 10 using Pspice, respectively, and the simulation results are shown in Figure 12.

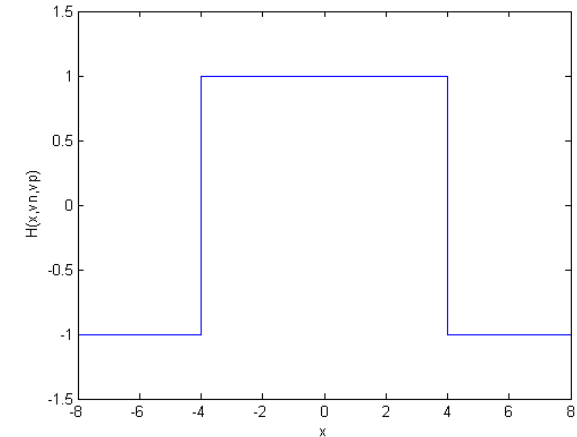

(a)

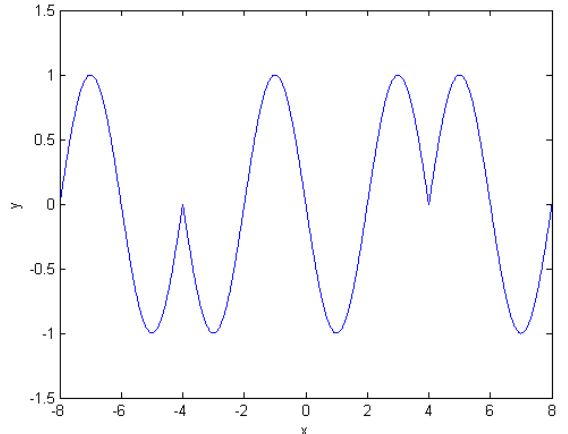

(b)

Figure 11. The output for $H\left(x, v_{n}, v_{p}\right)$ and $f(x)$ : (a) $H\left(x, v_{n}, v_{p}\right)=\operatorname{sign}(x+4)-\operatorname{sign}(x-4)-1$; (b) $f(x)=-\sin (0.5 \pi x) H(x,-4,4)$.

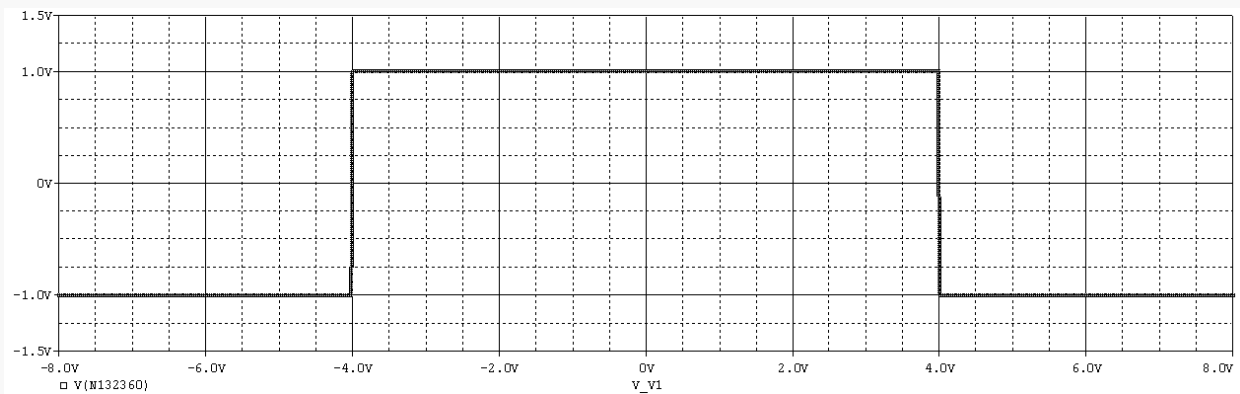

(a)

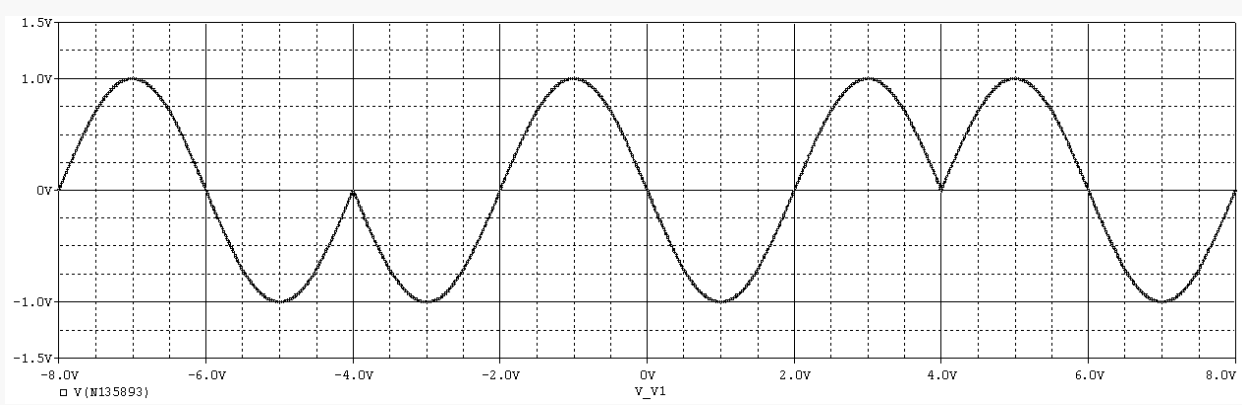

(b)

Figure 12. The output from circuit part N1 and N3: (a) $H\left(x, v_{n}, v_{p}\right)=\operatorname{sign}(x+4)-\operatorname{sign}(x-4)-1$; (b) $f(x)=-\sin (0.5 \pi x) H(x,-4,4)$. 
According to circuit theory, the circuit equations of the circuit diagram in Figure 10 can be obtained as follows:

$$
\left\{\begin{array}{l}
R_{3} C_{1} \frac{d X}{d t}=\frac{R_{2}}{R_{1}} Y \\
R_{8} C_{2} \frac{d Y}{d t}=\frac{R_{7}}{R_{6}} Z \\
R_{14} C_{3} \frac{d Z}{d t}=-\frac{R_{13}}{R_{11}} \frac{R_{16}}{R_{15}} Z-\frac{R_{13}}{R_{12}} \frac{R_{10}}{R_{9}} Y-\frac{R_{13}}{R_{20}} \frac{R_{5}}{R_{4}} f(x)
\end{array}\right.
$$

When the values of circuit components are chosen as: $C_{1}=C_{2}=C_{3}=1 u F, R_{3}=R_{8}=R_{14}=100 k$, $R_{5}=R_{10}=R_{16}=3 k$, and the other resistors equal to $10 k$, then Equation (18) is equivalent to Equation (19). $R_{3} C_{1}, R_{8} C_{2}$, and $R_{14} C_{3}$ are integration constants for the output voltage $X, Y$, and $Z$, respectively, and the frequency can be changed by setting different values for $R_{3} C_{1}, R_{8} C_{2}$, and $R_{14} C_{3}$.

$$
\left\{\begin{array}{l}
\frac{d X}{d t}=10 Y \\
\frac{d Y}{d t}=10 Z \\
\frac{d Z}{d t}=10(-0.3 Z-0.3 Y-0.3 f(x))
\end{array}\right.
$$

Changing the value of circuit component $R_{5}$, the other circuit components remain unchanged, the circuit Equation (18) is equivalent to Equation (3) with $a=c=0.3$ and $d$ is varied. In Figure 10, $v_{p}$ and $v_{n}$ correspond to $k_{p} / b$ and $-k_{n} / b$ in Equation (4), respectively. Evidently, $v_{p}$ must be less than the saturation voltage $v_{\text {cet }}$ of the operational amplifier $U_{10}$ in Figure 10 , and $v_{n}$ must be higher than the negative saturation voltage $-v_{c e t}$ of the operational amplifier $U_{11}$ in Figure 10, then following conditions must be satisfied.

$$
\left\{\begin{array}{l}
v_{p}=k_{p} / b<v_{c e t} \\
v_{n}=-k_{n} / b>-v_{c e t}
\end{array}\right.
$$

Assuming $k_{\max }=\max \left\{k_{p}, k_{n}\right\}$, the following inequality can be obtained:

$$
b>k_{\max } / v_{c e t}
$$

In the SFSF, the values of the parameters $k_{n}$ and $k_{p}$ are set equal to the scroll number of system (3) in the left and right part of the $x-y$ plane, respectively. Once the scroll number of the chaotic attractor is determined, the value of the parameter $b$ must satisfy Equation (21).

Let $v_{p}=12 \mathrm{~V}$ and $v_{n}=-12 \mathrm{~V}$, when $R_{5}=1.2 \mathrm{k} \Omega$, the circuit simulation result of system (3) in cycle state is shown in Figure 13a; when $R_{5}=3.5 k \Omega$, 6-scroll attractors are generated by system (3) are shown in Figure 13b.

Let $R_{5}=3.5 \mathrm{k} \Omega$ and the other electronic components remain unchanged, the comparative voltage $v_{p}$ and $v_{n}$ are selected as four cases, case 1: $v_{n}=-4 \mathrm{~V}, v_{p}=4 \mathrm{~V}$; case 2: $v_{n}=0 \mathrm{~V}, v_{p}=12 \mathrm{~V}$; case 3: $v_{n}=-4 V, v_{p}=12 \mathrm{~V}$, and case 4: $v_{n}=-12 \mathrm{~V}, v_{p}=8 \mathrm{~V}$. Then 2-scroll, 3-scroll, 4-scroll, and 5-scroll chaotic attractors are generated and shown in Figure 14.

Comparing Figure 6 with Figures 9 and 13 with Figure 14, we obtained that the results of circuit simulation by Pspice are consistent with that of numerical simulation by Matlab. The results in Figures 13 and 14 confirm that the chaotic attractors with different scroll numbers can be generated by system (3) with the SFSF of Equation (4) under appropriate system parameter values.

In multi-scroll chaotic system, nonlinear function is the key for generating scrolls. The complexity of circuit implementation varies with the construction of nonlinear functions. Some nonlinear functions are shown in Table 1. In Table 1, only signal function [32] gives the expression for generating $2 \mathrm{~N}$-scroll chaotic attractors, and the others give the expressions for generating $2 \mathrm{~N}$-scroll and $(2 \mathrm{~N}+1)$-scroll chaotic attractors. The number of the used electronic components in circuit realization of these nonlinear functions is presented in Table 1. $N_{s}, N_{A}, N_{R}, N_{V}$, and $N_{s w}$ represent the number of operational amplifiers, resistors, comparison voltage sources and switches, respectively. 


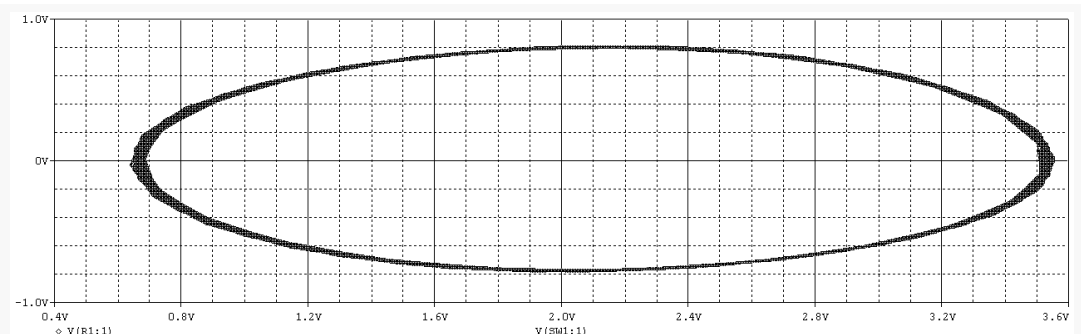

(a)

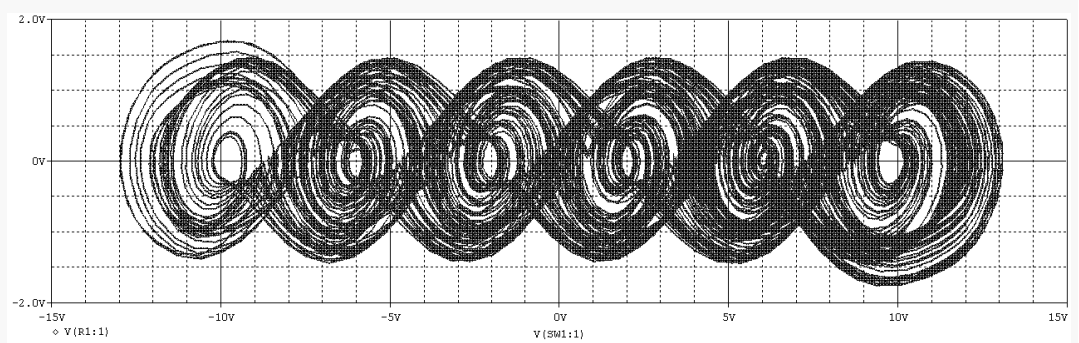

(b)

Figure 13. Different chaotic states are generated by electronic circuits with $v_{p}=12 \mathrm{~V}$ and $v_{n}=-12 \mathrm{~V}$ in Figure 10: (a) cycle state with $R_{5}=1.2 k \Omega$; (b) 6-scroll chaotic attractors with $R_{5}=3.5 k \Omega$.

Table 1. The number of used electronic components in circuit realization.

\begin{tabular}{ccccccc}
\hline Nonlinear Function Type & Function & $N_{S}$ & $N_{A}$ & $N_{R}$ & $N_{V}$ & $N_{S W}$ \\
\hline \multirow{2}{*}{ Triangular wave [31] } & $f_{11}(x)$ & $2 \mathrm{~N}$ & $\mathrm{~N}+2$ & $5 \mathrm{~N}+4$ & 0 & $\mathrm{~N}$ \\
& $f_{12}(x)$ & $2 \mathrm{~N}+1$ & $\mathrm{~N}+3$ & $5 \mathrm{~N}+9$ & 0 & $\mathrm{~N}+1$ \\
\hline Signal function [32] & $f_{2}(u)$ & $2 \mathrm{~N}$ & $2 \mathrm{~N}+1$ & $2 \mathrm{~N}+1$ & $2 \mathrm{~N}+1$ & $\mathrm{~N}+1$ \\
\hline \multirow{2}{*}{ Triangular wave [35] } & $f_{31}(u)$ & $2 \mathrm{~N}+1$ & $2 \mathrm{~N}+1$ & $6 \mathrm{~N}+2$ & $2 \mathrm{~N}$ & $\mathrm{~N}$ \\
& $f_{32}(u)$ & $2 \mathrm{~N}+2$ & $2 \mathrm{~N}+2$ & $6 \mathrm{~N}+6$ & $2 \mathrm{~N}+2$ & $\mathrm{~N}$ \\
\hline \multirow{2}{*}{ Adjustable sawtooth wave [44] } & $f_{41}(x)$ & $2 \mathrm{~N}+2$ & $2 \mathrm{~N}+1$ & $4 \mathrm{~N}+3$ & $2 \mathrm{~N}+1$ & $2 \mathrm{~N}+1$ \\
& $f_{42}(x)$ & $2 \mathrm{~N}+1$ & $2 \mathrm{~N}$ & $4 \mathrm{~N}+1$ & $2 \mathrm{~N}$ & $2 \mathrm{~N}$ \\
\hline \multirow{2}{*}{ Adjustable triangular wave [44] } & $f_{51}(x)$ & $2 \mathrm{~N}+2$ & $2 \mathrm{~N}+1$ & $6 \mathrm{~N}+4$ & $2 \mathrm{~N}+1$ & $2 \mathrm{~N}+1$ \\
& $f_{52}(x)$ & $2 \mathrm{~N}+1$ & $2 \mathrm{~N}$ & $6 \mathrm{~N}+1$ & $2 \mathrm{~N}$ & $2 \mathrm{~N}$ \\
\hline \multirow{2}{*}{ SFSF } & $f_{6}(x)$ & $2 \mathrm{~N}$ & 3 & 0 & 3 & 0 \\
& & $2 \mathrm{~N}+1$ & 3 & 0 & 3 & 0 \\
\hline
\end{tabular}

In Table 1, the equations of different nonlinear functions are described as follows:

$$
\begin{gathered}
f_{11}(x)=x+\sum_{n=0}^{2 N-1}(-1)^{n}\left[|x+(2 n-1)|-\sum_{n=1}^{N-1}|x-(2 n-1)|\right] \\
f_{12}(x)=x+\sum_{n=1}^{2 N}(-1)^{n-1}[|x+(2 n-1)|-|x-(2 n-1)|] \\
f_{31}(u)=\frac{1}{2 a} \quad \sum_{\substack{m \\
m \neq-N}}^{N}\left[\left|u-\left(2 m-\frac{|m|}{m}\right)+a\right|-\left|\left(2 m-\frac{|m|}{m}\right)-a\right|\right]-u \\
m \neq 0
\end{gathered}
$$




$$
\begin{aligned}
& f_{32}(u)=\frac{1}{2 a} \sum_{m=-N}^{N+1}\left[\left|u-\left(2 m-\frac{|m|}{m}\right)+a\right|-\left|u-\left(2 m-\frac{|m|}{m}\right)-a\right|\right]-u-1 \\
& m \neq 0 \\
& f_{31}(x)=x+\frac{1}{2 a} \sum_{\substack{m=-N \\
m \neq 0}}^{N}\left[\left|u-\left(2 m-\frac{|m|}{m}\right)+a\right|-\left|\left(2 m-\frac{|m|}{m}\right)-a\right|\right]-u \\
& f_{32}(x)=x+\frac{1}{2 a} \sum_{\substack{m=-N \\
m \neq 0}}^{N+1}\left[\left|u-\left(2 m-\frac{|m|}{m}\right)+a\right|-\left|u-\left(2 m-\frac{|m|}{m}\right)-a\right|\right]-u-1 \\
& f_{41}(x)=A_{0} \operatorname{sgn}(x)+\sum_{i=1}^{N}\left[\frac{A_{i-1}+A_{i}}{2} \operatorname{sgn}\left(x-\frac{2}{B} \sum_{j=0}^{i-1} A_{j}\right)\right]+\sum_{i=1}^{N}\left[\operatorname{sgn}\left(x+\frac{2}{B} \sum_{j=0}^{i-1} A_{j}\right)\right]-B x \\
& f_{42}(x)=\sum_{i=1}^{N}\left\{\frac{A_{i-1}+A_{i}}{2} \operatorname{sgn}\left(x-\frac{1}{B}\left(2 \sum_{j=0}^{i-1} A_{j}-A_{0}\right)\right]\right\}+\sum_{i=1}^{N}\left\{\frac{A_{i-1}+A_{i}}{2} \operatorname{sgn}\left(x+\frac{1}{B}\left(2 \sum_{j=0}^{i-1} A_{j}-A_{0}\right)\right]\right\}-B x \\
& f_{51}(x)=\sum_{n=-N}^{N} \frac{A_{n}}{2 a_{n}}\left[\left|\left(x-\frac{2 A_{n}}{B}\right)+a_{n}\right|-\sum_{n=1}^{N-1}\left|\left(x-\frac{2 A_{n}}{B}\right)-a_{n}\right|\right]-B x \\
& f_{52}(x)=\sum_{\substack{n=-N \\
n \neq 0}}^{N} \frac{A_{n}}{2 a_{n}}\left[\left|\left(x-\frac{A}{B}\left(2 n-\frac{|n|}{n}\right)\right)+a_{n}\right|-\left|\left(x-\frac{A}{B}\left(2 n-\frac{|n|}{n}\right)\right)+a_{n}\right|-B x\right. \\
& f_{6}(x)=\left\{\begin{array}{l}
-\sin (2 \pi b x),-k_{n} / b \leq x \leq k_{p} / b \\
+\sin (2 \pi b x), x>k_{p} / b \\
+\sin (2 \pi b x), x<-k_{n} / b
\end{array}\right.
\end{aligned}
$$

From Table 1, it can be seen that the SFSF has the same expression for generating $2 \mathrm{~N}$-scroll and ( $2 \mathrm{~N}$ +1 )-scroll chaotic attractors, but the others have different expressions. Furthermore, Figure 14 indicates that the SFSF regulates the number of scrolls only by adjusting the comparison voltages, and the rest nonlinear functions in Table 1 adjust the number of scrolls by controlling the on or off state of the switches in circuits. Table 1 shows that the circuit realization of the SFSF only needs three operational amplifiers and three comparison voltage sources, the others need operational amplifiers, resistors, switches, and comparison voltage sources. Moreover, the number of the electronic components of the SFSF is independent of the number of scrolls, while the others are related to the scroll number. Hence, the circuit realization of the SFSF is much simpler, more reliable than the rest ones in Table 1 , and the circuits of the SFSF for adjusting the number of scroll are much easier, especially, when the number of scrolls is large. Although the SFSF has many advantages for generating multi-scroll chaotic attractors, but its circuit realization need a sine signal generator, which cannot be implemented by discrete electronic components or operational amplifiers. Trigonometric wave function generator chip AD639 can generate sine signal, but it is out of production, so for hardware implementation of the SFSF at present, the problem of sine signal generator needs to be solved, which is what we're thinking about. 


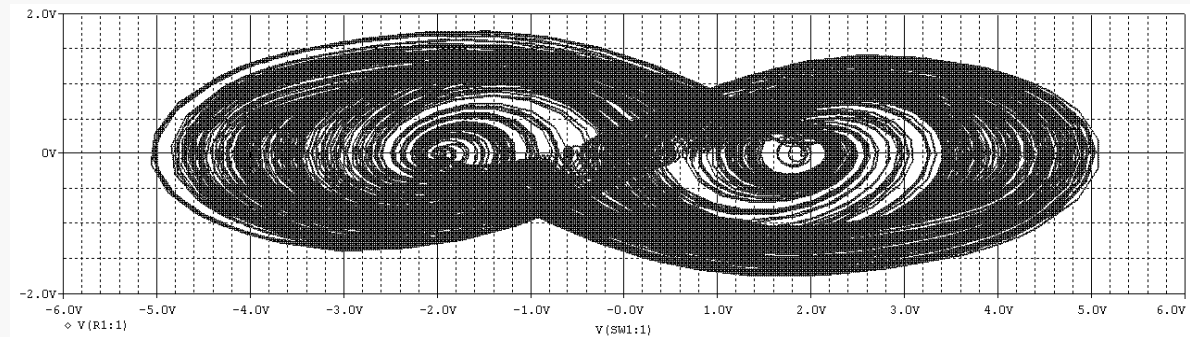

(a)

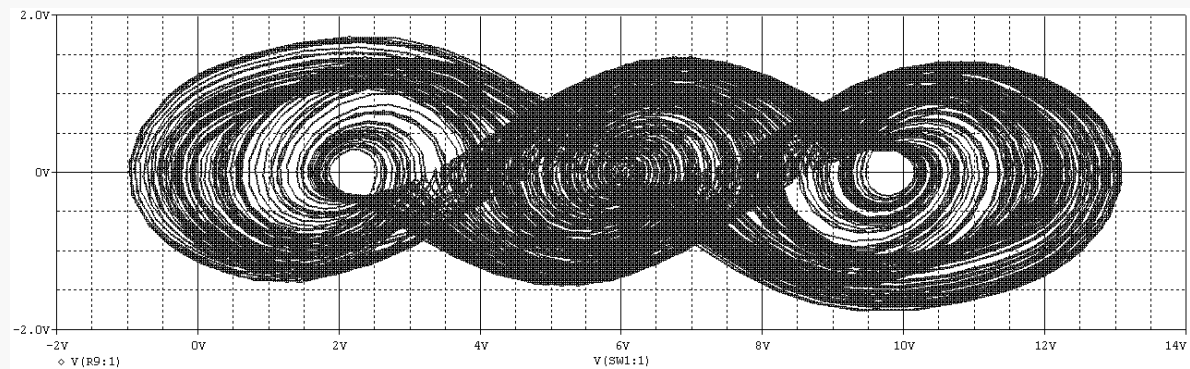

(b)

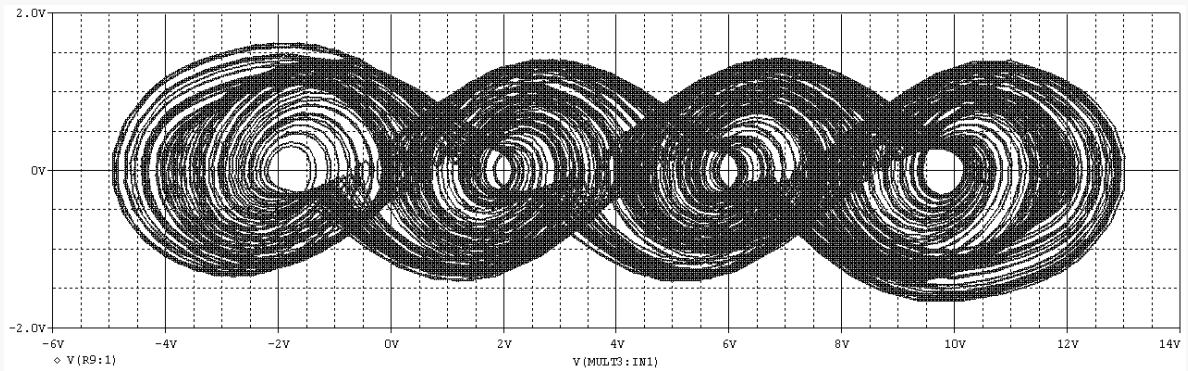

(c)

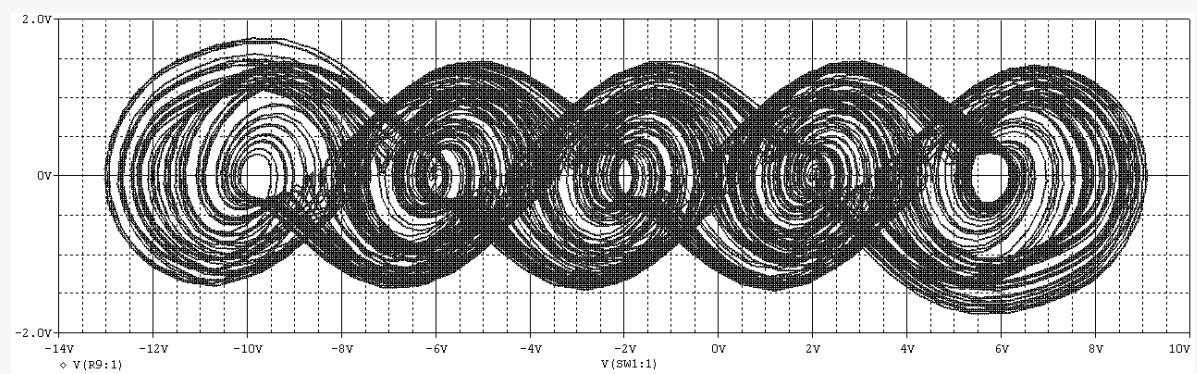

(d)

Figure 14. Chaotic attractors with different scroll number are generated with transient period about 10,000 unit time: (a) 2-scroll with $v_{p}=4(V)$ and $v_{n}=-4(V)$; (b) 3-scroll with $v_{p}=8(V)$ and $v_{n}=-4(V) ;(\mathbf{c}) 4$-scroll with $v_{p}=12(V)$ and $v_{n}=-4(V) ;\left(\right.$ d) 5-scroll with $v_{p}=8(V)$ and $v_{n}=-12(V)$.

\section{Conclusions}

This paper introduced a new chaotic system with nonlinearity of SFSF for generating multi-scroll chaotic attractors. Some dynamical properties of this system are investigated, such as equilibrium points, Lyapunov exponents, bifurcation diagrams, and chaotic phase diagrams. For verifying the availability and applicability of this system, an electronic circuit is designed using Pspice, the circuit simulation results are in good agreement with the numerical ones.

In contrast to other chaotic systems, this system with the SFSF has these unique advantages: (i) The number of the used electronic components in circuit realization is independent of the number of scrolls; (ii) the scrolls generated by this system can be distributed in the left half plane and/or the right 
half plane of the $x-y$ plane; (iii) the scroll number of the system in the left and right part of the $x-y$ plane can be arbitrarily adjusted by changing only two comparison voltages.

Author Contributions: Conceptualization, X.F. and P.D.; methodology, X.F.; software, P.D.; validation, P.D. and X.F.; formal analysis, X.F. and P.D.; writing—original draft, P.D.; writing—review and editing, X.F. All authors have read and agreed to the published version of the manuscript.

Funding: This research received no external funding.

Acknowledgments: We would like to thank the Editorial board and the anonymous reviewers.

Conflicts of Interest: The authors declare no conflict of interest.

\section{References}

1. Lorenz, E.N. Deterministic nonperiodic flow. J. Atmos. Sci. 1963, 20, 130-141. [CrossRef]

2. Chen, G.R.; Ueta, T. Yet another chaotic attractor. Int. J. Bifurc. Chaos 1999, 9, 1465-1466. [CrossRef]

3. Lu, J.H.; Chen, G.R. A new chaotic attractor coined. Int. J. Bifurc. Chaos 2002, 12, 659-661. [CrossRef]

4. Liu, C.X.; Liu, T.; Liu, L.; Liu, K. A new chaotic attractor. Chaos Solitons Fractals 2004, 22, 1031-1038. [CrossRef]

5. Bao, B.C.; Liu, Z.; Xu, J.P. New chaotic system and its hyperchaos generation. J. Syst. Eng. Electron. 2009, 20, 1179-1187.

6. Rossler, O.E. An equation for continuous chaos. Phys. Lett. A 1976, 57, 397-398. [CrossRef]

7. Sprott, J.C. Some simple chaotic flows. Phys. Rev. E 1994, 50, R647-R650. [CrossRef]

8. Liu, W.B.; Chen, G.R. A new chaotic system and its generation. Int. J. Bifurc. Chaos 2003, 13, 261-267. [CrossRef]

9. Tigan, G.; Opriş, D. Analysis of a 3D chaotic system. Chaos Solitons Fractals 2008, 36, 1315-1319. [CrossRef]

10. Zhou, W.N.; Xu, Y.H.; Lu, H.Q.; Pan, L. On dynamics analysis of a new chaotic attractor. Phys. Lett. A 2008, 372, 5773-5777. [CrossRef]

11. Pham, V.T.; Volos, C.; Jafari, S.; Wei, Z.C.; Wang, X. Constructing a Novel No-Equilibrium Chaotic System. Int. J. Bifurc. Chaos 2014, 24, 1450073. [CrossRef]

12. Wang, G.Y.; Chen, D.J.; Lin, J.Y.; Chen, X. The application of chaotic oscillators to weak signal detection. IEEE Trans. Ind. Electron. 1999, 46, 440-444. [CrossRef]

13. Wang, G.Y.; He, S.L. A quantitative study on detection and estimation of weak signals by using chaotic Duffing oscillators. IEEE Trans. Circuits Syst. I Fundam. Theory Appl. 2003, 50, 945-953. [CrossRef]

14. Gokyildirim, A.; Uyaroglu, Y.; Pehlivan, I. A novel chaotic attractor and its weak signal detection application. Optik 2016, 127, 7889-7895. [CrossRef]

15. Li, Y.X.; Chen, X.; Yu, J.; Yang, X.H. A Fusion Frequency Feature Extraction Method for Underwater Acoustic Signal Based on Variational Mode Decomposition, Duffing Chaotic Oscillator and a Kind of Permutation Entropy. Electronics 2019, 8, 8010061. [CrossRef]

16. Lu, J.A.; Wu, X.Q.; Lu, J.H. Synchronization of a unified chaotic system and the application in secure communication. Phys. Lett. A 2002, 305, 365-370. [CrossRef]

17. Liu, L.Z.; Zhang, J.Q.; Xu, G.X.; Liang, L.S.; Wang, M.S. A chaotic secure communication method based on chaos systems partial series parameter estimation. Acta Phys. Sin. 2014, 63, 010501.

18. Pano-Azucena, A.D.; Jose, R.M.; Tlelo-Cuautle, E.; de Jesus Quintas-Valles, A. Arduino-based chaotic secure communication system using multi-directional multi-scroll chaotic oscillators. Nonlinear Dyn. 2017, 87, 2203-2217. [CrossRef]

19. Barakat, M.L.; Mansingka, A.S.; Radwan, A.G.; Salama, K.N. Generalized Hardware Post-processing Technique for Chaos-Based Pseudorandom Number Generators. ETRI J. 2013, 35, 448-458. [CrossRef]

20. Kaçar, S. Analog circuit and microcontroller based RNG application of a new easy realizable 4D chaotic system. Optik 2016, 127, 9551-9561. [CrossRef]

21. Vaidyanathan, S.; Akgul, A.; Kaçar, S.; Çavuşoğlu, U. A new 4-D chaotic hyperjerk system, its synchronization, circuit design and applications in RNG, image encryption and chaos-based steganography. Eur. Phys. J. Plus 2018, 133, 11872-11878. [CrossRef]

22. Haroun, M.F.; Gulliver, T.A. Real-time image encryption using a low-complexity discrete 3D dual chaotic cipher. Nonlinear Dyn. 2015, 82, 1523-1535. [CrossRef] 
23. Xie, E.Y.; Li, C.Q.; Yu, S.M.; Lü, J.H. On the cryptanalysis of Fridrich's chaotic image encryption scheme. Signal Process. 2017, 132, 150-154. [CrossRef]

24. Hua, Z.Y.; Jin, F.; Xu, B.X.; Huang, H.J. 2D Logistic-Sine-coupling map for image encryption. Signal Process. 2018, 149, 148-161. [CrossRef]

25. Kaur, M.; Kumar, V. Efficient image encryption method based on improved Lorenz chaotic system. Electron. Lett. 2018, 54, 562-563. [CrossRef]

26. Asgari-Chenaghlu, M.; Balafar, M.A.; Feizi-Derakhshi, M.R. A novel image encryption algorithm based on polynomial combination of chaotic maps and dynamic function generation. Signal Process. 2019, 157, 1-13. [CrossRef]

27. Voss, H.U. Anticipating chaotic synchronization. Phys. Rev. E. 2000, 61, 5115-5119. [CrossRef]

28. Ravelo, B.; Wan, F.Y.; Feng, J. All-Pass Negative Group Delay Function with Transmission Line Feedback Topology. IEEE Access 2019, 7, 155711-155723. [CrossRef]

29. Ravelo, B.; Wan, F.Y.; Ge, J. Anticipating Actuator Arbitrary Action with a Low-Pass Negative Group Delay Function. IEEE Trans. Ind. Electron. 2020, 1-9. [CrossRef]

30. Suykens, J.A.K.; Vandewalle, J. Generation of $n$-double scrolls $(n=1,2,3,4, \ldots)$. IEEE Trans. Circuits Syst. IFundam. Theory Appl. 1993, 40, 861-867. [CrossRef]

31. Zhang, C.X.; Yu, S.M. Design and implementation of a novel multi-scroll chaotic system. Chin. Phys. B 2009, 18, 119-129.

32. Wang, F.Q.; Liu, C.X. A new multi-scroll chaotic generator. Chin. Phys. 2007, 16, 942-945.

33. Liu, X.Z.; Shen, X.M.; Zhang, H.T. Multi-scroll chaotic and hyperchaotic attractors generated from Chen system. Int. J. Bifurc. Chaos 2012, 22, 1250033. [CrossRef]

34. Chen, D.Y.; Sun, Z.T.; Ma, X.Y.; Chen, L. Circuit implementation and model of a new multi-scroll chaotic system. Int. J. Circuit Theory Appl. 2014, 42, 407-424. [CrossRef]

35. Luo, X.H.; Tu, Z.W.; Liu, X.R.; Cai, C.; Liang, Y.L.; Gong, P. Implementation of a novel two-attractor grid multi-scroll chaotic system. Chin. Phys. B 2010, 19, 070510.

36. Chen, Z.; Wen, G.L.; Zhou, H.A.; Chen, J.Y. A new M x N-grid double-scroll chaotic attractors from Rucklidge chaotic system. Optik 2017, 136, 27-35. [CrossRef]

37. Zhang, G.T.; Wang, F.Q. A novel multi-scroll chaotic generator: Analysis, simulation, and implementation. Chin. Phys. B 2018, 27, 018201. [CrossRef]

38. Lü, J.H.; Han, F.L.; Yu, X.H.; Chen, G.R. Generating 3-D multi-scroll chaotic attractors: A hysteresis series switching method. Automatica 2004, 40, 1677-1687. [CrossRef]

39. Peng, Z.P.; Wang, C.H.; Luo, X.W. A novel multi-directional multi-scroll chaotic system and its CCII+ circuit implementation. Optik 2014, 125, 6665-6671. [CrossRef]

40. Wang, C.H.; Xu, H.; Yu, F. A novel approach for constructing high-order Chua's circuit with multi-directional multi-scroll chaotic attractors. Int. J. Bifurc. Chaos 2013, 23, 13500223. [CrossRef]

41. Deng, W.H.; Lü, J.H. Generating multi-directional multi-scroll chaotic attractors via a fractional differential hysteresis system. Phys. Lett. A 2007, 369, 438-443. [CrossRef]

42. Zhang, Y.; Yu, S.M.; Liu, M.H. Generating multi-scroll hyperchaotic attractors based on FPGA technology. J. Circuits Syst. 2007, 12, 39-43.

43. Tlelo-Cuautle, E.; Rangel-Magdaleno, J.J.; Pano-Azucena, A.D.; Obeso-Rodelo, P.J.; Nunez-Perez, J.C. FPGA realization of multi-scroll chaotic oscillators. Commun. Nonlinear Sci. Numer. Simul. 2015, 27, 66-80. [CrossRef]

44. Yu, S.M.; Lü, J.H.; Leung, H.; Chen, G.R. Design and implementation of n-scroll chaotic attractors from a general jerk circuit. IEEE Trans. Circuits Syst. I Regul. Pap. 2005, 52, 1459-1476.

45. Yalcin, M.E. Multi-scroll and hypercube attractors from a general jerk circuit using Josephson junctions. Chaos Solitons Fractals 2007, 34, 1659-1666. [CrossRef]

46. He, S.B.; Sun, K.H.; Wang, H.H.; Ai, X.X.; Xu, Y.X. Design of n-dimensional multi-scroll Jerk chaotic system and its performance. J. Appl. Anal. Comput. 2016, 6, 1180-1194.

47. Ma, J.; Wu, X.Y.; Chu, R.T.; Zhang, L.P. Selection of multi-scroll attractors in Jerk circuits and their verification using Pspice. Nonlinear Dyn. 2014, 76, 1951-1962. [CrossRef]

48. Tchitnga, R.; Nguazon, T.; Fotso, P.H.L.; Gallas, J.A.C. Chaos in a Single Op-Amp-Based Jerk Circuit: Experiments and Simulations. IEEE Trans. Circuits Syst. II Express Briefs 2016, 63, 239-243. [CrossRef] 
49. Tang, W.K.S.; Zhong, G.Q.; Chen, G.; Man, K.F. Generation of n-scroll attractors via sine function. IEEE Trans. Circuits Syst. I Fundam. Theory Appl. 2001, 48, 1369-1372. [CrossRef]

50. Hu, X.; Liu, C.; Liu, L.; Ni, J.K.; Li, S.L. Multi-scroll hidden attractors in improved Sprott A system. Nonlinear Dyn. 2016, 86, 1725-1734. [CrossRef]

51. Luo, X.H. Circuitry implementation of a novel nonautonomous hyperchaotic Liu system based on sine input. Chin. Phys. B 2009, 18, 3304-3308.

52. Hu, X.Y.; Liu, C.X.; Liu, L.; Yao, Y.P.; Zheng, G.C. Multi-scroll hidden attractors and multi-wing hidden attractors in a 5-dimensional memristive system. Chin. Phys. B 2017, 26, 110502. [CrossRef]

53. Chen, Z.; Wen, G.L.; Zhou, H.A.; Chen, J.Y. Generation of grid multi-scroll chaotic attractors via hyperbolic tangent function series. Optik 2017, 130, 594-600. [CrossRef]

54. Gunay, E.; Altun, K. Multi-Scroll Chaotic Attractors in SC-CNN via Hyperbolic Tangent Function. Electronics 2018, 7, 7050067. [CrossRef]

55. Wang, Z.; Volos, C.; Kingni, S.T.; Azar, A.T.; Pham, V.T. Four-wing attractors in a novel chaotic system with hyperbolic sine nonlinearity. Optik 2017, 131, 1071-1078. [CrossRef]

56. Signing, V.R.F.; Kengne, J.; Pone, J.R.M. Antimonotonicity, chaos, quasi-periodicity and coexistence of hidden attractors in a new simple 4-D chaotic system with hyperbolic cosine nonlinearity. Chaos Solitons Fractals 2019, 118, 187-198. [CrossRef]

57. Tlelo-Cuautle, E.; Quintas-Valles, A.D.J.; Fraga, G.D.L.; Rangel-Magdaleno, J.D.J. VHDL Descriptions for the FPGA Implementation of PWL-Function-Based Multi-Scroll Chaotic Oscillators. PLoS ONE 2016, 11, 0168300. [CrossRef]

58. Ali, A.M.; Ramadhan, S.M.; Tahir, F.R. A Novel 2D-Grid of Scroll Chaotic Attractor Generated by CNN. Symmetry 2019, 11, 99. [CrossRef]

59. Chen, L.P.; Pan, W.; Tenreiro Machado, J.A.; Lopes, A.M.; Wu, R.C.; He, Y.G. Design of fractional-order hyper-chaotic multi-scroll systems based on hysteresis series. Eur. Phys. J. Spec. Top. 2017, 226, 3775-3789. [CrossRef]

60. Lü, J.H.; Chen, G.R.; Yu, X.H.; Leung, H. Design and analysis of multiscroll chaotic attractors from saturated function series. IEEE Trans. Circuits Syst. I Regul. Pap. 2004, 51, 2476-2490. [CrossRef]

61. Zhang, X.; Wang, C.H. A Novel Multi-Attractor Period Multi-Scroll Chaotic Integrated Circuit Based on CMOS Wide Adjustable CCCII. IEEE Access 2019, 7, 16336-16350. [CrossRef]

62. Chen, S.B.; Zeng, Y.C.; Xu, M.L.; Chen, J.S. Construction of grid multi-scroll chaotic attractors and its circuit implementation with polynomial and step function. Acta Phys. Sin. 2011, 60, 020507.

63. Wang, C.H.; Luo, X.W.; Wan, Z. Generation and circuit implementation of multi-block multidirectional grid multi-scroll chaotic attractors. Optik 2014, 125, 6716-6721. [CrossRef]

64. Rajagopal, K.; Çiçek, S.; Naseradinmousavi, P.; Khalaf, A.J.M.; Jafari, S.; Karthikeyan, A. A novel parametrically controlled multi-scroll chaotic attractor along with electronic circuit design. Eur. Phys. J. Plus 2018, 133, 354. [CrossRef]

65. Wang, Z.H.; Cang, S.J.; Ochola, E.O.; Sun, Y.X. A hyperchaotic system without equilibrium. Nonlinear Dyn. 2012, 69, 531-537. [CrossRef]

66. Sanchez-Lopez, C. Automatic synthesis of chaotic attractors. Appl. Math. Comput. 2011, 217, 4350-4358. [CrossRef]

(C) 2020 by the authors. Licensee MDPI, Basel, Switzerland. This article is an open access article distributed under the terms and conditions of the Creative Commons Attribution (CC BY) license (http://creativecommons.org/licenses/by/4.0/). 